\title{
Anastomosis entre el nervio cubital y el nervio mediano: reporte de caso
}

\author{
Martín Alonso Gonzales Nolasco ${ }^{1,2}$
}

\section{RESUMEN}

El trayecto del nervio mediano desciende del plexo braquial colocándose en el codo por delante de la epitróclea reposicionándose luego al centro del antebrazo. Durante su recorrido es común la ausencia de ramos comunicantes nerviosos con el nervio cubital. Este reporte describe el hallazgo de una conexión nerviosa entre el nervio mediano y cubital dando pie a la recolección de información sobre la relación entre dichos nervios. Las implicancias clínicas de la presencia de dicha conexión nerviosa van de la mano con sintomatología y el diagnóstico diferencial de los síndromes que afectan a los nervio mediano y cubital tanto en el antebrazo como en muñeca o mano.

Este hallazgo se realizó durante los trabajos de disección en el Anfiteatro de Anatomía Humana de la Universidad Nacional Mayor de San Marcos (Perú). Se reporta esta comunicación nerviosa utilizando la información disponible resaltando su importancia quirúrgico-clínico.

Palabras clave: Nervio Mediano, Nervio Cubital, Conexión, Variación

\section{Anastomosis between the ulnar nerve and the median nerve: case report}

\begin{abstract}
The path of the median nerve descends from the brachial plexus to the elbow, it is positioned in front of the epitroclea and then repositioned on the central axis of the forearm. During this path, it is common to find the absence of communicative branches between the median nerve and the ulnar nerve. This case report describes the finding of a nerve communicative branch between this and ulnar nerve which gives rise to the collection of information on the relationship between this nerves. The clinical implications of the presence of said nerve connection go hand in hand with symptomatology and the differential diagnosis of syndromes that affect the median and ulnar nerves in both the forearm and wrist or hand.

This finding was made during the 2015 dissection work at the human anatomy amphitheater of the National University of San Marcos (Peru). This connection is reported using the available information highlighting its importance in the surgical-clinical field.
\end{abstract}

Key words: Median Nerve, Ulnar nerve, Connection, Variation.

\section{Introducción}

El nervio mediano y el nervio cubital se origina en el plexo braquial (1) descendiendo ambos al codo para posicionarse delante de la epitróclea y en el canal epitrocleo-olecraneano respectivamente (2). El nervio mediano se coloca posterior al músculo flexor común superficial de los dedos y separado del músculo flexor común profundo de los dedos por tejido conjuntivo (3). En el codo y antebrazo, este nervio origina ramas nerviosas hacia las articulaciones y a los músculos del compartimiento anterior del antebrazo (4).

1. Facultad de Medicina "San Fernando", Universidad Nacional Mayor de San Marcos, Lima, Perú.

2. Sociedad de Ciencias Básicas de San Fernando.

Citar como : Gonzales Nolasco MA. Anastomosis entre el nervio cubital y el nervio mediano: reporte de caso. Rev Peruana de Morfologia. 2021;

2(1):33-37, doi: https//doi.org/1051343/revperuanamorfologia.v2i1.409

Recibido: 2020-12-18; Aceptado: 2021-04-18

Autor corresponsal: Martín Alonso Gonzales Nolasco; Email: alonso10medicina@gmail.com; Dirección: Av. Argentina 6148 Dpto 1 - Callao 
En referencia a esta región anatómica los tratados clásicos de anatomía como Rouviere (1924) y García Porto Carrero (2005), no refieren conexiones constantes o destacadas entre el nervio mediano y cubital (5).

Sin embargo, las conexiones entre el nervio cubital y el nervio mediano han sido documentadas por pocos estudiosos desde el siglo XVIII y XIX entre las que destacan las realizadas por Martín (1763) y Gruber (1870), de ahí que las conexiones nerviosas a nivel de antebrazo entre el nervio Mediano y Cubital se les denomina Anastomosis de Martin-Gruber (AMG) (6) las cuales pueden salir tanto del tronco principal del nervio mediano como del nervio interóseo anterior el cual es ramo del nervio mediano (6).

Estudios más recientes electromiográficos como el de Fenicel (1976) mencionó que hasta un $20 \%$ de pacientes pueden tener una distribución y/o comunicación atípica entre ambos nervios(7).

Abayev (2005) nos menciona que la incidencia de la AMG a nivel del tercio superior del antebrazo puede estar en el rango del $15-20 \%(8)$.

La presencia de esta comunicación nerviosa podría ser explicada porque ambos nervios se desarrollan a partir de una misma región embrionaria (9).

Sobre este aspecto, Herrera y col. (2009) refieren, en base a conjeturas anteriores, que la aparición de la AMG se le podría catalogar como un vestigio filogenético ya que esta rama tiene cierta homología con el tronco nervioso ventral común que inerva a los músculos flexores de la extremidad superior de algunas especies como los primates(10). Sin embargo, Luokas Marios y cols. (2011) proponen que estas conexiones aparecen en ocasiones cuando hay ausencia de conexión entre el nervio cubital y mediano a nivel de la región palmar(11).

El interés médico involucra la descripción de patologías que afectan al nervio mediano y cubital. En parte se puede deber, según Oswald (1998), porque en los casos donde aparece la $\mathrm{AMG}$, las fibras motoras nacen del tronco nervioso anterior (ramo nervioso típica del mediano) y llega al nervio cubital como vía de acceso para los músculos intrínsecos de la mano, lo que genera que axones motores de músculos inervados por el nervio cubital tengan un nacimiento desde el nervio mediano(12).

Al respecto, Herrera(10) mencionó el carácter asintomático de la aparición de la AMG; sin embargo, esto dificultaría el diagnóstico diferencial del síndrome del túnel del carpo y el síndrome del canal cubital del carpo, lo que ameritaría exámenes electrofisiológicos más específicos. Para demostrar ello, Nakashima (1993) indicó que la estimulación electrofisiológica del nervio mediano a nivel del codo genera un potencial de acción tanto en la región hipotenar (inervada por el nervio cubital), en la región Tenar (inervada por el Nervio Mediano) y en el primer músculo interóseo dorsal de la mano (13).

Sobre lo antes mencionado, Shu y cols. (1999) mencionaron que la AMG generaría pérdidas motoras atípicas de lesiones periféricas, lo que se presume pueda ser la explicación de los diagnósticos erróneos de dichas lesiones. Con referencia al Síndrome del Túnel carpiano, también nos corrobora la permanencia del tono muscular de la región tenar cuando se presenta dicha anastomosis. Shu nos indica que el conocimiento de la comunicación entre nervio cubital y mediano puede prevenir el lesionar estos nervios periféricos(14).

A continuación, se describirá el reporte de una variación anatómica del nervio mediano a nivel superior del antebrazo encontrado en una muestra cadavérica de miembro superior izquierdo, durante la disección en el Anfiteatro de Anatomía Humana de la Facultad de Medicina de "San Fernando" de la Universidad Nacional Mayor de San Marcos.

\section{Disección cadavérica}

El hallazgo anatómico se realizó durante la disección de un espécimen de miembro superior izquierdo como parte de los trabajos de disección en el Anfiteatro de Anatomía Humana de la Facultad de Medicina de "San Fernando" de la Universidad Nacional Mayor de San Marcos.

El nervio cubital, luego de pasar por el canal epitrócleoolecraneano, pasa a situarse en el antebrazo justo debajo del músculo flexor cubital del carpo de manera solitaria en el tercio proximal del músculo y luego en el resto del musculo acompañado por la arteria cubital hasta llegar a la articulación de la muñeca.

El nervio mediano atraviesa la fosa cubital y se sitúa en la región media del antebrazola región media del antebrazo (Fig. 1, -5-). Luego sigue su recorrido en una posición central hasta llegar a la articulación de la muñeca donde atraviesa el túnel del carpo para llegar a la palma de la mano.

Poco después de salir de la fosa cubital, el nervio mediano emite un ramo nervioso que cursa por debajo del músculo flexor cubital del capo en dirección inferomedial (Fig. 2 -5-) y se llega a fusionar con el nervio cubital estableciendo 
una comunicación directa entre ambas ramas terminales del plexo braquial.

Para la correcta exposición de la conexión nerviosa se procedió a disecar el músculo pronador redondo y afianzar por medio de sutura nylon una porción de fibras musculares del músculo flexor cubital del carpo, el cual, presentaba una pequeña porción desinsertada en su extremo proximal.

Además, se observan ciertas fibras nerviosas que nacen del nervio mediano mientras este atraviesa la fosa cubital y que terminan inervando los extremos laterales de los fascículos del Músculo Flexor Cubital del Carpo (Fig. 2)

Los detalles topográficos de las variaciones en arterias y venas fueron examinados, registrados y fotografiados.

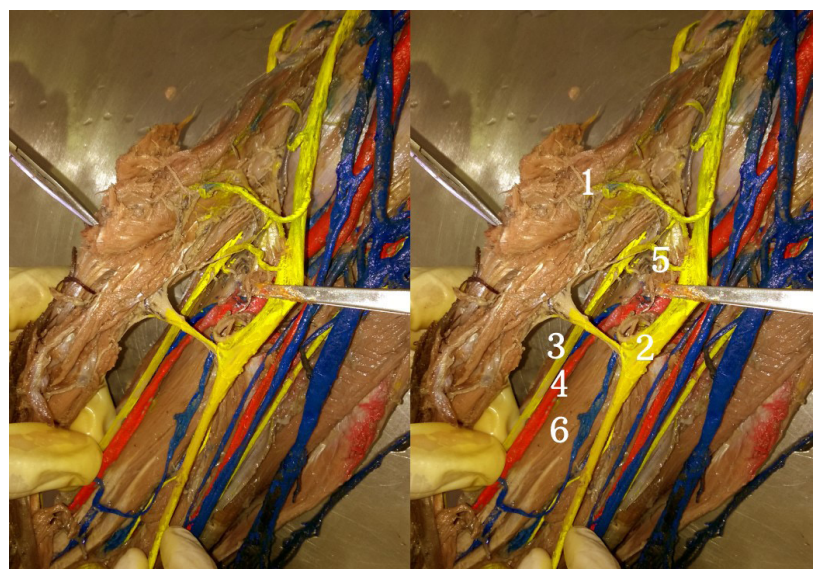

Fig. 1. Descripción anatómica de la anastomosis nerviosa: 1. Músculo Flexor Cubital del Carpo. 2. Nervio Mediano. 3. Nervio Cubital. 4. Arteria Cubital. 5. Anastomosis nerviosa entre el nervio mediano y nervio cubital a nivel proximal del antebrazo. 6. Músculo Flexor Superficial Común de los Dedos.

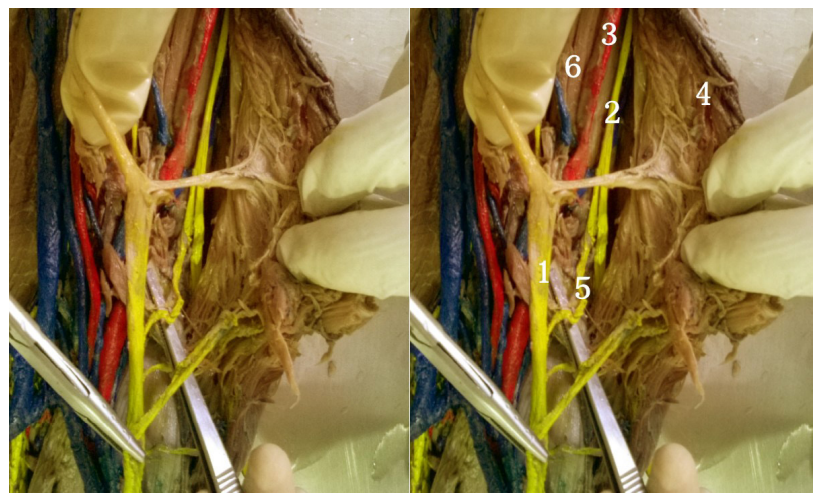

Fig 2. Descripción anatómica de la anastomosis nerviosa: 1.Nervio Mediano. 2. Nervio Cubital. 3. Arteria Cubital. 4. Músculo Flexor Cubital del Carpo. 5. Anastomosis nerviosa entre el nervio cubital y el nervio mediano. 6. Músculo Flexor Superficial Común de los dedos.

\section{DISCUSIÓN}

En la muestra cadavérica del presente reporte, se encontró en el miembro superior izquierdo la AMG que nació del nervio mediano en el tercio proximal del antebrazo y llegó al nervio cubital en un nivel más inferior del que se posicionan los músculos epitrocleares. Para lograr una disección adecuada se tuvo que separar los fascículos musculares de los músculos de la primera capa muscular del antebrazo (músculo flexor cubital del carpo, músculo palmar largo y músculo pronador redondo).

Sobre la localización, Almeida y col (1999) demostraron, en disecciones realizadas en 40 antebrazos, que las AMG se encuentran en el plano entre los músculos epitrocleares y el músculo flexor común profundo de los dedos (15). Ellos encontraron 5 casos (12.5\%) con presencia de conexiones en antebrazo; en 3 casos dichas conexiones se encontraron en el miembro izquierdo, (15) de forma similar a este reporte de caso en el cual la AMG se encontró a nivel del antebrazo en el miembro superior izquierdo.

Con referencia a su distribución, Luciana y col. (2003), mediante la disección de 64 regiones anteriores de antebrazo en muestras cadavéricas, encontraron la AMG en un $7.8 \%$ de las muestras ( 5 casos) y reportó un solo caso donde la variación fue bilateral en un mismo cadáver. De los 5 casos mencionados, en 4 casos la AMG se originan desde el nervio interóseo anterior (ramificación del nervio mediano) mientras que en 1 solo caso se origina del tronco principal del nervio mediano (16). La AMG encontrada en nuestra muestra cadavérica se originó del tronco principal del nervio mediano y fue unilateral

En cuanto a las características sociodemográficas, muchos de estos estudios cadavéricos no se encontró alguna diferencia significativa entre razas y/o entre sexos $(16,17)$. La muestra anatómica de este reporte de caso fue un espécimen aislado de miembro superior sin datos especificados de género y edad.

Con respecto al trayecto, nuestra miembro disecado evidencia que la raíz anastomótica en el nervio cubital se presentaba en la intersección entre el tercio superior y tercio medio del antebrazo presentando un trayecto oblicuo en dirección inferomedial. Sobre este trayecto, Kasakos y cols. (2005) encuentran como una posible explicación del curso transverso u oblicuo de la AMG el cual dependía de si el extremo anastomótico con el nervio cubital se encontraba en el tercio superior o tercio medio del antebrazo (18).

Analizando la longitud, la AMG que presentó la nuestra 
pieza cadavérica es de $3.9 \mathrm{~cm}$ aprox., medida que se encuentra dentro del rango longitud establecido por Taams en Sudáfrica (1997) quien en 56 cadáveres encontró un promedio de distancia del origen es $6 \mathrm{~cm}$ distal del epicóndilo medial del húmero (con rango variable entre $2.5 \mathrm{~cm}$ a $8 \mathrm{~cm}$ ). El promedio de longitud media de las comunicaciones nerviosas fue de $3.8 \mathrm{~cm}$ (con rango de 2.5 a $5.5 \mathrm{~cm})(17)$.

Con lo ya descrito, surge la necesidad de poder agrupar las diversas presentaciones de la AMG; en favor de ello, Erdem y col. (2002) confeccionaron una clasificación de las comunicaciones nerviosas entre el nervio cubital y el nervio mediano tomando en cuenta los músculos que inervan: "El tipo I donde el nervio mediano está involucrado en la inervación de los músculos hipotenares, el tipo II que inerva los músculos interóseos dorsales, y el tipo III, los músculos tenares". En sus estudios, Erdem encontró la AMG en 27 de los 100 sujetos, y halló con mayor frecuencia el tipo 11 (19). Basándonos en esta clasificación vemos la imposibilidad de poder determinar con certeza el tipo al que pertenece nuestro reporte de caso al no contar con implementos electromiográficos adecuados.

Sobre esta última clasificación mencionada, se resalta que las clasificaciones de la AMG discrepan entre sí debido a la falta de una denominación unificada y los distintos puntos de referencia para clasificarlos como su origen o número de ramas comunicantes (10). Como alternativa a estas formas de observar dicha anastomosis, Sarikcioglu (2006) propone agregar términos adicionales "aferente y eferente" en referencia a la dirección de las fibras nerviosas para lograr un entendimiento internacional en la descripción anatómica (20).
Como queda demostrado en estudios y descripciones clínicas $(10,13)$, las patologías que pueden afectar al nervio mediano podrían a su vez generar sintomatología atípica por la presencia de estas conexiones nerviosas descritas.

\section{CONCLUSIONES}

Las conexiones nerviosas entre el nervio mediano y cubital son poco usuales, pero, su consideración nos permite ser más precavidos a la hora de realizar procedimientos quirúrgicos en zonas como el antebrazo. Este caso resalta el interés de conocer las conexiones entre el nervio mediano y el nervio cubital por la sintomatología que generan las patologías que afecta individualmente a alguno de estos nervios donde, debido a la presencia de la anastomosis, podría presentar síntomas alterados o incompletos.

Esto representa un aspecto a tomar en cuenta por parte de los médicos, especialmente a los traumatólogos y anestesiólogos, pues las anestesias aplicadas a ciertos ramos nerviosos no tendrían efecto al tener dicha zona una doble inervación sensitiva.

\section{Agradecimiento}

A la estudiante de medicina Ana Lida Brañez Condorena por su contribución a la redacción del presente artículo

\section{Fuente de Financiación}

Autofinanciado

\section{Conflicto de Interés}

El autor manifiesta que no existe ningún tipo de conflicto de interés en el presente trabajo.

\section{Contribución del autor}

El autor diseñó el estudio, recopiló e interpretó los datos para el trabajo, participó en la redacción del manuscrito y aprobó su versión final.

\section{REFERENCIAS}

1. Testut L, Latarjet A. Tratado de Anatomía Humana. 9na edición. Barcelona: Editorial Salvat Editores SA; 1986.P.272.

2. Testut L, Latarjet A. Tratado de Anatomía Humana. 9na edición. Barcelona: Editorial Salvat Editores SA; 1986.P.273.

3. Rouviére H, Delmas A. Anatomía Humana, descriptiva, topográfica y funcional. 11va edición. Barcelona: Editorial Masson SA; 2005.p.256.

4. Testut L, Latarjet A. Tratado de Anatomía Humana. 9na edición. Barcelona: Editorial Salvat Editores SA; 1986.P.277.

5. Rouviére H, Delmas A. Anatomía Humana, descriptiva, topográfica y funcional. 11va edición. Barcelona: Editorial Masson SA; 2005.p. 199.
6. Salazar VR. Inervación anómala del antebrazo y mano: anastomosis entre el nervio mediano y cubital. Rev Med Cos Cen. 2012;69(600):31-35.

7. Iyer V, Fenichel GM. Normal median nerve proximal latency in carpal tunnel syndrome: a clue to coexisting Martin-Gruber anastomosis. J Neurol Neurosurg Psychiatry. 1976;39(5):449-452.

8. Abayev B, Ha E, Cruise C. Ulnar dominant hand and forearm: an electrophysiologic approach. Neurologist. 2005;11(5):294-300.

9. Felippe, MM. Telles, FL. Soares, ACL. Felippe, FM. Anastomosis between median nerve and ulnar nerve in the forearm. Journal of Morphological Sciences. 2012;29(1):2326.
10. Herrera E, Anaya C, Abril AM, Lozano WM, Avellaneda YC, Cruz AM. Revisión de Tema. Revista Salud UIS. Universidad Industrial de Santander. 2009;41:157-168.

11. Loukas M, Abel N, Tubbs RS, Matusz P, Zurada A, Cohen-Gadol AA. Neural interconnections between the nerves of the upper limb and surgical implications. J Neurosurg. 2011;114(1):225-35.

12. Oswald TA. Anatomic considerations in evaluation of the proximal ulnar nerve. Phys Med Rehabil Clin N Am. 1998;9(4):777-94.

13. Nakashima T. An anatomic study on the Martin-Gruber anastomosis. Surg Radiol Anat. 1993;15(3):193-5.

14. Shu HS, Chantelot C, Oberlin C, Alnot JY, Shao H. Martin-Gruber communicating 
Branch: anatomical and histological study. Surgical and Radiologic anatomy. 1999;21:115-118.

15. de Almeida JA, Vitti M, Garbino JA. Estudo anatômico da anastomose de Martin-Grub. Revista Hansenologia Insternationalis. 1999;24(1):15-20.

16. Prates LC, de Carvalho VM, Prates JC, Langone F, Marretto Esquisatto MA. The Martin-Gruber anastomosis in brasilinaz: an anatomical study. Brazilian Journal of Morpological Sciences. 2003;20(3):177180.
17. Taams KO. Martin-Gruber connections in South Africa. An anatomical study. J Hand Surg Br. 1997;22(3):328-30.

18. Kazakos KJ, Smyrnis A, Xarchas KC, Dimitrakopoulou A, Verettas DA. Anastomosis between the median and ulnar nerve in the forearm. An anatomic study and literature review. Acta Orthop Belg. 2005;71(1):29-35.

19. Erdem HR, Ergun S, Erturk C, Ozel S. Electrophysiological evaluation of the incidence of martin-gruber anastomosis in healthy subjects. Yonsei Med J. 2002;43(3):291-5.
20. Sarikcioglu L, Demirel BM. Martin-Gruber and Marinacci communications--anatomic or physiologic consideration. J Hist Neurosci. 2006;15(2):99-101. 